\title{
Dossier « Territoires en transition environnementale » Introduction. Quand les territoires font face aux nouveaux
enjeux de l'environnement
}

\author{
Pierre-Henri Bombenger ${ }^{1}$, Corinne Larrue ${ }^{2}$ \\ 1 Aménagement et sciences de l'environnement, Haute école d'ingénierie et de gestion du Canton de Vaud, \\ Haute école spécialisée de Suisse Occidentale, 1401 Yverdon-les-Bains, Suisse ${ }^{1}$ \\ 2 Aménagement et urbanisme, Institut d'Urbainisme de Paris, 77454 Marne-la-Vallée cedex 2, France ${ }^{2}$
}

Ce dossier est issu d'un colloque organisé en décembre 2011 par l'UMR Citeres (CNRS-université de Tours) en partenariat avec l'université de Strasbourg, l'Université Laval et l'Université du Québec à Montréal, intitulé « Territoire et environnement: des représentations à l'action ». Sur la base d'un bilan des transformations apparues dans la construction et la mise en pratique des représentations de l'espace au regard des enjeux environnementaux, la problématique générale de ce colloque visait à aborder la question des transformations réciproques des pratiques territoriales par la prise en compte des questions environnementales et des effets de ces pratiques spatiales, et de leur aménagement, sur l'environnement.

Les communications présentées ici traitent principalement des effets des nouveaux enjeux environnementaux liés aux changements climatiques, analysés au prisme de la notion de territoire. Comme le montrent ces quatre contributions, que ce soit en matière d'atténuation (réduction des émissions de gaz à effet de serre par le développement d'énergies alternatives comme l'éolien) ou d'adaptation (ajustement des systèmes naturels ou humains afin d'atténuer les effets néfastes ou d'exploiter des opportunités bénéfiques du changement climatique), le changement climatique et ses politiques associées viennent (re)questionner le cadrage territorial des politiques environnementales. Cette thématique permet plus particulièrement de mettre en tension des enjeux globaux, dont les références sont a-territoriales, et les enjeux locaux encastrés dans des systèmes d'action établis. En interrogeant les conditions à réunir pour que l'enjeu environnemental fasse l'objet d'une territorialisation par le local, voire qu'il constitue le fondement d'un projet territorial, ce dossier, à travers quatre études de cas, met en question la pertinence de la notion de territoire pour appréhender les politiques climatiques/énergétiques.

Deux principales questions peuvent être proposées en introduction à ces lectures. D'une part, il ressort de ces analyses une réelle difficulté pour ces nouvelles problématiques environnementales à s'intégrer dans les dispositifs territoriaux mis en place au cours des cinquante dernières années pour traiter des problématiques environnementales (1). D'autre part, ces communications pointent que l'action publique associée à ces nouveaux enjeux environnementaux vient recomposer au moins partiellement les systèmes argumentatifs des systèmes d'action territoriaux, sans pour autant changer les représentations héritées du territoire (2).

\footnotetext{
Auteur correspondant : P.-H. Bombenger, pierre-henri.bombenger@heig-vd.ch

1 Chargé de recherche en aménagement et sciences de l'environnement à l'Institut G2C et chercheur associé à l'UMR CNRS 7324 Citeres, de l'université de Tours, équipe COST.

2 Professeure en aménagement de l'espace à l'Institut d'urbanisme de Paris, chercheur à l'UMR CNRS 7324 Citeres, de l'université de Tours, équipe COST.
} 


\section{Quand l'environnement interpelle la notion de territoire}

Depuis les années 1960, les enjeux environnementaux se sont progressivement mariés avec les modes d'action des territoires, de même qu'avec leurs projets (Zuindeau, 2010). La montée en puissance des enjeux environnementaux dans les années 1960-1970 s'est rapidement accompagnée d'une appropriation par les acteurs locaux, en recherche de bénéfices pour leur territoire : les externalités positives, le marketing vert, la qualité de vie ont constitué autant de motifs de valorisation des ressources environnementales des territoires (Melé et Larrue, 2008 ; Gumuchian et Pecqueur, 2007 ; Faure et Douillet, 2005).

Par ailleurs, durant les années 1980-1990, la mise en œuvre des politiques environnementales a mis en exergue la capacité de rejet, par les acteurs locaux, de projets $\mathrm{s}$ 'inscrivant dans ces politiques : on trouve ainsi de nombreux exemples de conflits localisés sur les thématiques des déchets ou de la protection de la nature et du cadre de vie au regard de multiples infrastructures (Melé, 2013 ; Kirat et Torre, 2008). Ces mouvements ont également montré l'importance de la mobilisation locale comme modalité de mise en œuvre des politiques environnementales associées à des projets considérés comme dommageables. Le cadre territorial constituait alors un rempart vis-à-vis de l'implantation de ces objets de politique publique, bénéficiant à d'autres espaces que ceux impactés.

Avec les années 2000-2010, on voit poindre de nouvelles problématiques liées à des enjeux plus globaux : le changement climatique constitue aujourd'hui la pierre angulaire de ces enjeux, mais la question des continuités écologiques pose également des problèmes similaires ${ }^{3}$. Ces nouveaux enjeux environnementaux se caractérisent par la dimension globale de leurs cadres de référence « et une inscription des choix dans une perspective planétaire » (F. Bertrand et E. Richard dans ce dossier).

Ces enjeux, que l'on peut qualifier de nouveaux au regard de l'évolution des problématiques environnementales, viennent interroger les cadres territoriaux de l'action publique, et partant la notion de territoire jusque-là largement mobilisée par les politiques environnementales territoriales. Deux principaux enseignements peuvent être mis en exergue à la lecture de ces communications. D'une part, la difficile pertinence de la notion de territoire pour encadrer l'action publique face

\footnotetext{
3 Cette thématique fait actuellement l'objet du projet de recherche Erudie (2012-2015), « Les espaces ruraux au défi des infrastructures écologiques ", coordonné par P.-H. Bombenger et C. Larrue, et financé dans le cadre du programme Diva du ministère de l'Écologie, du Développement durable et de l'Énergie.
}

à ces nouveaux enjeux environnementaux : de quelle manière ces nouveaux enjeux s'encastrent-ils dans des processus territoriaux en place ? D'autre part, les articles montrent bien qu'au-delà de ces difficultés, on assiste néanmoins à des processus de territorialisation, mais qui restent spécifiques et encore largement expérimentaux.

Ainsi, les quatre articles présentés dans ce dossier permettent de remettre en question la capacité des systèmes d'action territoriaux à s'emparer de ces nouveaux enjeux environnementaux.

Le texte de F. Bertrand et E. Richard montre bien la difficulté de s'approprier localement les enjeux du changement climatique: seule l'impulsion réglementaire nationale, via les schémas régionaux climat air énergie (SRCAE) et les plans climat territoriaux (PCET) rendus obligatoires pour les régions et certaines collectivités par la loi Grenelle 2 (loi n ${ }^{\circ} 2010-788$ du 12 juillet 2010 portant engagement national pour l'environnement), conduit à une timide et très homogène saisie de ces questions. Mais comme le notent justement ces auteurs, "sans que ces objectifs soient réellement territorialisés, c'est-à-dire ajustés aux spécificités locales ». Le "problème climat » reste encore à l'état de mise sur l'agenda local ou régional, via un processus descendant ( $\mathrm{du}$ niveau international au niveau local) sans pour autant faire l'objet d'une réelle saisie locale. Cette situation s'explique pour ce qui concerne les politiques d'atténuation, par une focale principalement axée vers les émetteurs individuels, bâtiments publics, industriels, domestiques ou agricoles. Du coup, la médiation par les territoires reste très faible d'autant que les compétences des collectivités dans ce domaine sont encore balbutiantes. Pour les politiques d'adaptation, et comme le montrent les premiers travaux en la matière (Bertrand et Rocher, 2013), là encore la saisie territoriale reste difficile. A. Nadai et $\mathrm{O}$. Labussière mettent en exergue l'absence "d'une prospective du milieu », que l'on peut élargir à l'absence d'une vision territoriale environnementale qui permettrait d'intégrer ces nouveaux enjeux au sein des projets de territoire.

De même, il ressort de la lecture croisée du texte d'A. Nadai et d'O. Labussière, et de celui de M.-J. Fortin et Y. Fournis, la nécessité d'un recadrage territorial des problèmes, car les enjeux traités ne s'insèrent pas dans les dispositifs existants : soit que le territoire de référence reste à construire $\mathrm{du}$ fait de la nouveauté de l'enjeu (dans le cas de l'éolien offshore notamment), soit que les processus de planification sont mal adaptés au projet comme dans le cas québécois. Par les conflits qu'ils ont générés, ces deux cas de mise en place de projets éoliens montrent bien cette inadéquation aux cadres existants. Les deux articles en détaillent les tenants. On verra toutefois, pour ce qui concerne les aboutissants, que ces tensions ont donné lieu in fine à des dispositifs innovants tant sur le plan juridique que sur le plan des processus de mise en œuvre des politiques publiques. 
Cette inadéquation entre dispositifs territoriaux préexistants et nouvel enjeu environnemental est également très claire dans le cas de la dépoldérisation (L. Goeldner-Gianella et F. Bertand). Ce projet apparaît en nette rupture avec les politiques territoriales en place jusqu'ici et fondées sur l'implication des « communautés territoriales » pour référer à l'expression proposée par M.-J. Fortin et Y. Fournis. Cela apparaît d'autant plus clairement que la dépoldérisation fait perdre aux espaces concernés leur qualité de territoire, tant d'un point de vue juridique que du point de vue fonctionnel (au regard des usages de l'espace). Dans ce cas, il ne s'agit pas uniquement d'une atteinte au territoire, par une pression environnementale plus forte, mais bien d'une remise en cause de l'existence physique - et sociale - de ce territoire en tant que tel. Ce qui revient à exacerber la question de l'acceptabilité sociale de ce projet sur laquelle nous reviendrons par la suite.

Ainsi, chacun à leur manière, les quatre articles posent la question de la pertinence de la notion de territoire pour encadrer l'action publique face à ces nouveaux enjeux environnementaux. Mais si l'on peut déceler des signes de décalage évident entre les cadres territoriaux d'action et ces nouvelles questions environnementales, pour autant cela ne fait pas disparaître totalement les processus de territorialisation de ces enjeux.

En effet, ce que mettent également en exergue ces contributions, ce sont les multiples modalités de territorialisation de l'action publique environnementale. Celleci procède d'une mobilisation simultanée des multiples échelles opérantes qu'induisent ces nouveaux objets. Les trois contributions portant sur les projets éoliens et sur la dépoldérisation montrent bien la complexité des processus en jeu. Dans ces trois exemples, les projets s'inscrivent dans des cadres extraterritoriaux et mettent en évidence les processus de mobilisation simultanée des différents niveaux de décision ou d'horizon. Si territorialisation il y a, c'est à travers de multiples allers-retours entre niveau local et niveau central, particulièrement évidents dans le cas de l'éolien offshore. Ces dynamiques complexes se caractérisent par des processus souples, ouverts, de pilotages multiples et précaires (M-J. Fortin et Y. Fournis). Ils se concrétisent via des tâtonnements, des expérimentations, forcées dans le cas de l'éolien offshore du fait de l'absence de référence, ou spontanées dans le cas de la dépoldérisation (où le processus «naturel » de submersion marine sert d'expérimentation pour d'autres espaces).

Plus généralement, les tensions entre gestion sectorielle et enjeux territoriaux pour ce qui concerne l'éolien offshore conduisent à des innovations procédurales et instrumentales que l'on peut interpréter comme autant de processus de territorialisation. Le bricolage est de mise, et ces processus en construction ne suivent pas (ou plus) de modèle de mise en œuvre, qu'il soit de coproduction ou de coconstruction (Duran et Thoenig, 1996). Il s'agit d'un mode d'intervention qu'il faut à chaque fois redéfinir. Il est fondé sur la négociation avec les usagers en place, sur la mobilisation de leurs savoirs (cas des pêcheurs) et surtout il ne se réalise pas à " environnement social constant » (A. Nadai et O. Labussière). L'attention aux compensations financières (cas de l'éolien offshore) ou aux retombées économiques et à leur capacité d'internalisation (cas de l'éolien québécois), qui relèvent de processus de territorialisation plus classiques, $n^{\prime}$ apparaît plus suffisante pour assurer une acceptabilité territoriale de ces projets.

Enfin, ce qui apparaît en filigrane de ces communications, c'est la tension encore importante entre les projets de territoire fondés sur les cadres d'action territoriaux en place (tant institutionnels que participatifs) et les enjeux environnementaux globaux. Cette tension repose sur une mutuelle ignorance qui devient source de conflits lorsqu'il y a rencontre. Les objectifs globaux annoncés de réduction des consommations énergétiques ou des gaz à effet de serre (GES) ne sont pas pensés dans une vision territorialisée. Une globalisation des solutions est mise en regard de la globalisation des problèmes. Or la résistance/médiation territoriale est néanmoins toujours incontournable au moment où s'amorce le processus de mise en œuvre. Ces articles mettent ainsi en évidence une médiation qui n'est plus activée au seul niveau local, mais qui conduit à des processus de mobilisation à différents niveaux et portant sur différents registres. Cela est d'autant plus vrai que, comme nous le verrons par la suite, le retrait de l'État dans l'accompagnement de l'aménagement des territoires, que ce soit en France ou au Québec, rend cette confrontation encore plus frontale.

\section{La question environnementale, pour quelles conceptions des territoires?}

Pour autant, face au constat d'une faible pertinence des cadres territoriaux d'action pour prendre en charge ces nouveaux enjeux environnementaux, ces derniers ne pourraient-ils pas être en mesure de recomposer, au moins partiellement, la conception de ces territoires ou de leurs projets?

Alors que l'environnement est souvent présenté comme un nouveau cadre de représentations des territoires, force est de constater que de manière prépondérante cet enjeu politique et social présente un paradoxe remarquable. S'il constitue le fondement d'un nombre important de tensions, de controverses, voire de conflits, autour de l'évolution d'un territoire - à tel point que certains observateurs argumentent que le conflit est inhérent à toute transformation des usages du sol et de l'environnement, et donc aux politiques et projets d'aménagement du territoire (Jobert, 1998) -, il ne génère 
que très occasionnellement une coalition de valeurs ou d'intérêts susceptible de produire un projet de territoire capable in fine de s'institutionnaliser. Les préoccupations environnementales largement diffusées dans les agendas politico-administratifs nationaux et internationaux peinent ainsi à fonder, à l'échelle locale, des instruments de mobilisation suffisamment puissants, pour structurer les trajectoires des territoires locaux, à travers un projet commun et dépasser les antagonismes que ces questions sociales génèrent dans les arbitrages entre préservation et valorisation des ressources environnementales.

Dans ce contexte, l'échelle locale apparaît comme une scène de légalisation territoriale de l'environnement - à travers les documents de planification du droit des sols et d'un affrontement afin de déterminer par qui et sur la base de quelles représentations sociales sont régulées ces dispositions. Dans tous les cas, les projets éoliens et de polders semblent générer de forts conflits locaux, mais sans constituer un nouveau référentiel de projet territorial local. Dans nos quatre cas, tout se passe comme si l'environnement était faiblement appropriable par le local, comme si la capacité de territorialisation des enjeux globaux restait limitée.

En effet, à l'échelle locale, l'environnement occupe encore souvent le rôle de support de légitimation de politiques sectorielles, largement permis par la diffusion de notions polysémiques comme le développement durable, la nouvelle gouvernance environnementale ou la gestion intégrée. Â l'exemple des cas de l'éolien au Québec et en France, ces projets n'apparaissent souvent que comme la mise en œuvre locale de politiques nationales à finalité énergétique, avant d'être des politiques environnementales. Leur attache au territoire local est quasi inexistante. Elle se réduit à une question de mise en cohérence des multiples dispositifs de planification du droit des sols, avec les usages de l'espace transformés par cette nouvelle activité énergétique. Les projets éoliens sont très éloignés, tout du moins dans leur processus d'élaboration et dans leurs finalités, des politiques de gestion des territoires ruraux, comme le sont les projets de parcs naturels régionaux, fondés - a priori - sur la valorisation des patrimoines endogènes. Dans le cas des développements éoliens présentés ici, le territoire est placé en position de support dont les principales qualités sont la force du vent et la capacité à porter les lignes électriques vers les centres urbains. La dimension environnementale, entendue dans un sens large, constitue tout à la fois un argument de légitimation de ces projets industriels à l'échelle globale - la réduction des émissions de GES et du risque nucléaire - et une source de tensions et de conflits à l'échelle locale - incidence paysagère, problèmes de cohabitation des usages du territoire et de l'exploitation de la ressource halieutique. Dans le cas de la dépoldérisation $\mathrm{du}$ bassin d'Arcachon, l'enjeu environnemental va à l'encontre du projet de territoire fondé notamment sur la valorisation agrotouristique des prairies et porté par le parc naturel régional et le projet de parc naturel marin. Si le cas des stratégies d'adaptation au changement climatique (F. Bertrand et E. Richard) semble plus consensuel, les auteurs soulignent que dans leur processus historique, ces politiques n'en sont encore qu'à leurs prémices et que les contraintes qu'elles font peser sur les usages du territoire sont encore faibles, voire positives pour les propriétaires des logements qui peuvent obtenir des aides publiques conséquentes pour en accroître l'efficacité énergétique. Si ces politiques deviennent plus impératives ${ }^{4}$, les oppositions vont nécessairement devenir plus nombreuses.

Autrement dit, dans ces quatre cas d'étude, l'environnement est une notion fondamentalement définie par de multiples référentiels propres aux individus et groupes qui s'affrontent pour imposer leurs objectifs, leurs valeurs, leurs représentations sociales. Ces environnements non consensuels font l'objet de luttes pour définir les acceptions légitimes et dominantes au sein du projet, mais pas nécessairement avec les acteurs territoriaux. Les accords et les coalitions noués entre acteurs de nos cas d'études sont insuffisamment robustes pour construire le socle d'un projet de territoire. Les acteurs de ces projets jouent sur des scènes très différentes (nationales/locales pour le cas du projet éolien de Veulettes-sur-Mer, internationales/locales pour le cas de la filière éolienne québécoise) et les ressources de ces projets sont souvent extérieures au local (des multinationales de l'énergie pour l'éolien, des ministères pour l'expertise de dépoldérisation, des organismes internationaux pour l'adaptation au changement climatique). Mais ces acteurs locaux n'ont accès qu'à l'une ou l'autre des multiples scènes de négociation que ces projets complexes mettent en lumière. Ils apparaissent quelque peu démunis face aux ressources d'action dont disposent les porteurs de projets précités en matière de moyens financiers, mais également de réseaux et d'expertises. Cette inégalité de ressources explique également les difficultés d'appropriation des projets par ces acteurs.

Faiblement appropriés localement au point de ne pas pouvoir réellement parler de territoire (Melé et Larrue, 2008), les espaces où se jouent ces projets environnementaux pourraient apparaître tout simplement comme les périmètres d'action des administrations ou des porteurs de projet chargés de la régulation de ces dispositifs. Or ce n'est pas réellement le cas, dans la mesure où la nature relativement nouvelle des questions posées, sans dispositif ou instrument d'accompagnement incitatif, organisationnel ou coercitif préconçu - les dispositifs et outils

\footnotetext{
4 Comme c'est le cas pour la loi sur l'énergie de l'État de Vaud en Suisse, entrée en vigueur progressivement le $1^{\mathrm{er}}$ juillet 2014, et qui impose que les nouvelles constructions doivent couvrir au moins $20 \%$ de leurs besoins en électricité par une énergie renouvelable produite sur site.
} 
sont testés en même temps que le projet, cela est particulièrement visible dans le cas de Veulettes-sur-Mer - rend assez incertaine l'existence d'un territoire d'action cohérent. Contrairement à des politiques construites autour d'un projet spatialisé - par exemple un parc naturel régional ou une zone Natura 2000 -, il semble que dans les quatre projets exposés dans ce dossier, l'enjeu sectoriel (énergétique et/ou climatique) rende secondaire la question du territoire. En outre, cette faible territorialité des dispositifs, remplacés par de multiples scènes parallèles (tantôt locales, tantôt entre les ministères dans le cas éolien offshore) et des périmètres variables, limite la capacité d'implication des acteurs locaux et place les protagonistes dans un cadre d'incertitude sur les effets de ces projets (juridiques et écologiques dans le cas de l'éolien offshore) et sur leur efficacité face à l'enjeu environnemental en question (dans le cas de la dépoldérisation et de l'adaptation au changement climatique). Au final, les retombées territoriales apparaissent minimes, incertaines, voire opposées aux trajectoires de développement prises par ces territoires. Ainsi, le projet de dépoldérisation pourrait aboutir à la disparition du territoire concerné.

La question de l'acceptabilité sociale de ces projets notamment pour les cas éoliens et de dépoldérisation interroge également la capacité des États à définir des politiques procédurales (Lascoumes, 1996) pour lesquelles ils ne possèdent pas - ou plus - les ressources d'ingénierie territoriale de mise en œuvre. Si les politiques de l'environnement, tant en France qu'au Québec, ont longtemps été l'apanage des ministères et de leurs services déconcentrés, elles procèdent de manière croissante par appels à projets ${ }^{5}$, sur le modèle de l'expérimentation-auto-évaluation à l'échelle territoriale. Le contenu organisationnel de ces politiques tend à imposer des attentes et des engagements généraux exogènes - notamment européens et internationaux sur la question de la réduction des émissions des GES et de l'adaptation aux conséquences du changement climatique - par le droit, mais dont les instruments peinent à trouver une accroche locale. Territorialiser le droit dans un contexte d'incertitude sur les effets du phénomène à réguler (la perte de biodiversité par les corridors écologiques, l'adaptation au changement climatique sans disposer de modèles microrégionaux des effets territoriaux de ces évolutions, etc.), tout en tenant compte des exigences d'usagers du territoire qui recourent de manière croissante au contentieux juridique sur les questions d'aménagement du territoire (Jacq, 2009) et d'une diminution des moyens d'ingénierie territoriale (Bombenger, 2011) semble ainsi un défi qui ne peut qu'aboutir à des compromis territoriaux boiteux. Or cet enjeu de la perte

\footnotetext{
5 Cas actuel de la politique des « paysages humanisés » portée par le ministère du Développement durable, de l'Environnement et des Parcs du Québec.
}

d'ingénierie publique est autant présent en France qu'au Québec. Les mesures mises en œuvre dans le cadre des politiques de « rationalisation de l'action publique » ont abouti à perdre aussi bien la connaissance d'usage des territoires que la capacité d'expertise scientifique et technique de l'État et de ses agences pour la suppléer par un recours à l'expertise privée. Mais cette pratique généralisée depuis une décennie omet que l'autorité d'une connaissance et d'un savoir relève autant de sa rationalité scientifique et technique que de l'acteur qui l'apporte - et la porte-dans le processus décisionnel, particulièrement dans un cadre d'aménagement du territoire conflictuel, où l'incertitude est forte et les recours juridiques des parties prenantes croissants. Ainsi, dans nos quatre études de cas, les collectifs légitimes pour définir l'acception localement acceptable de l'environnement dans le territoire ne sont pas suffisamment robustes pour pouvoir imposer leurs vues.

Au final, l'émergence de ces nouveaux enjeux et l'incapacité à les réguler avec les outils existants induisent un double déplacement. D'une part, la lecture de ces nouveaux enjeux globaux se fait au prisme des usages locaux existants. Mais d'autre part, il apparaît surtout que les tensions, voire les conflits, qui naissent de cette confrontation entre héritages et déplacements dans les usages du territoire introduits par les questions énergétiques/climatiques ne génèrent pas les fondements d'une alternative crédible pour faire émerger un projet de territoire. Dans ce contexte, il devient alors intéressant de questionner les facteurs et processus qui permettraient d'intégrer pleinement les enjeux énergétiques et climatiques comme fondements d'un projet collectif de territoire, et partant d'accroître l'implication des usagers $\mathrm{du}$ territoire dans la gestion de ces nouveaux enjeux afin d'en tirer parti pour le développement local.

\section{Références}

Bombenger, P.-H., 2011. L'urbanisme en campagne. Pratiques de planification des sols et d'aide à la décision dans des communes rurales françaises. Thèse de doctorat en Aménagement et en Sciences de l'environnement, Université François-Rabelais et UQAM, Tours et Montréal (online: http://www.archipel. uqam.ca/4492/1/D2275.pdf).

Bertrand, F., Rocher, L., 2013. Les territoires face aux changements climatiques: une première génération d'initiatives locales, Éditions Peter Lang, Coll. EcoPolis.

Duran, P., Thoenig, J.-C., 1996. L'État et la gestion publique territoriale, Revue Française de Science Politique, 46, 4, 580-623.

Faure, A., Douillet, A.-C., 2005. L'Action publique et la question territoriale, Grenoble, Presses universitaires de Grenoble.

Gumuchian, H., Pecqueur, B., 2007. La Ressource territoriale, Paris, Economica, Anthropos.

Jacq, P., 2009. La judiciarisation de la vie publique locale, in Bidégaray, C., Cadiou, S., Pina, C., L'Élu local aujourd'hui, Grenoble, Presses universitaires de Grenoble, 81-94.

\section{(1)}


Jobert, A., 1998. L'aménagement en politique ou ce que le syndrome NIMBY nous dit de l'intérêt général, Politix, 42, 67-92.

Kirat, T., Torre, A., 2008. Territoires de conflits: analyses des mutations de l'occupation de l'espace, Paris, L'Harmattan.

Lascoumes, P., 1996. Rendre gouvernable : de la «traduction» au « transcodage ». L'analyse des processus de changement dans les réseaux d'action publique, in Centre universitaire de recherches administratives et politiques, La Gouvernabilité, Paris, Presses universitaires de France, 325-338.

Melé, P., 2013. Conflits de proximité et dynamiques urbaines, Rennes, Presses universitaires de Rennes.

Melé,P., Larrue, C., 2008. Territoires d'action, Paris, L'Harmattan, Coll. Itinéraires géographiques.

Zuindeau, B., 2010. Développement durable et territoire, Villeneuve d'Ascq, Presses universitaires du Septentrion, 411-420. 